\title{
MECHANICAL PROPERTIES OF SELFEXPANDABLE STENTS
}

\author{
Josef Hanuš, Jiř́ Záhora
}

Charles University in Prague, Faculty of Medicine in Hradec Králové, Czech Republic: Department of Medical Biophysics

\begin{abstract}
Summary: The stents or stentgrafts (covered stents) are the medical instruments used in invasive radiology for miniinvasive treatment of stenosis and aneurisms especially in the blood circulation system. We measured and compared the mechanical parameters of different selfexpandable Nitinol stents, which differed in the geometry (radius and length), in the type of construction (number of branches and rising of winding) and in the diameter of the used wire. The results of measurements confirmed the theoretical assumptions that just the diameter of the Nitinol wire significantly influences the rigidity and the level of compressibility of the stent as well. The compromise must be found between the required rigidity of the stent and the minimal size of the delivery system. The exact description of the relation between the mechanical properties and geometry and construction of the stents enables us to design the stent to fit the patient's needs. The results of measurement are also necessary for the design and identification of the parameters of the models of the stents.
\end{abstract}

Key words: Stent; Mechanical parameters; Strain-stress curve

\section{Introduction}

The miniinvasive implantation of stents or stentgrafts for various indications is a very quickly developed sophisticated trend in medicine $(1,2)$. These methods are used for correction of aneurysms or stenosis in the cardiovascular system, for correction of limited passage in the digestive system, in the urinary tract or in the biliary duct. The minimal traumatization of the patient, the improvement of the life comfort, shorten of the patient's stay in the hospital are the typical advantages of these methods. But on the opposite side also some disadvantages can occur, typically the leakage or the shift of stent, limited chemical resistance of the materials of the stents. We suppose that the main reason of these problems is that the mechanical properties, dimension and the dynamical properties of the stent do not correspond to the properties of the vessel or generally of tissue where this stent is implanted. That is the reason, why we want to identify and to describe these relations between the mechanical properties of stents and tissues. Our originally developed device for measurement of mechanical properties of stents or vessels and results of measurements of selfexpandable Nitinol stents and short reference to their chemical resistance are described in this paper.

\section{Chemical resistance}

The stents are designed for the long time implantation so that the chemical interaction with the inner environment of the human body is expected. This long time interaction (at least 10 years) is substituted by experiments that simulate the expected corrosion in the real time. The electro chemical degradation tests (according to ISO/DIS 1099315/2000) were applied for the evaluation of the resistance of Nitinol and steel wires, the typical material for the construction of stents. The special solution of electrolytes simulates the inner environment of the body, in our case the blood plasma+oxygen, but also the artificial saliva can be used. The level of corrosion of different material is given in the figures 1 (laboratories of Technical University of Ostrava). There are many parameters that characterize the chemical resistance of materials but the pictures are the most illustrative for the first information. Results show the different corrosion level of tested material that depends not only
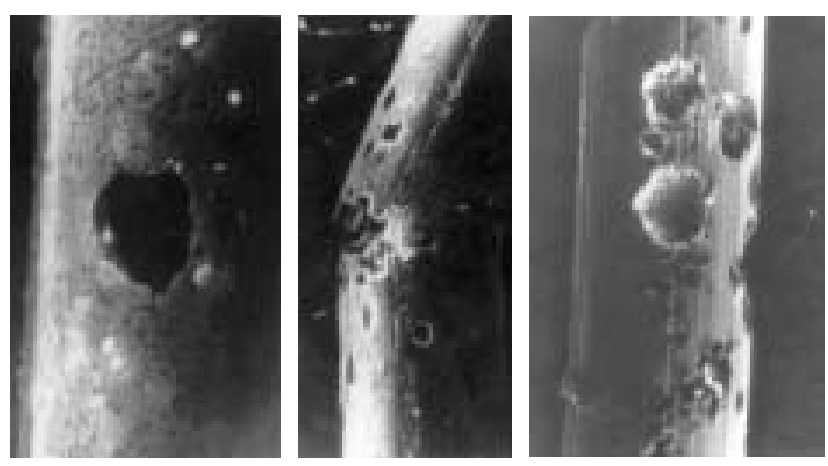

Fig. 1: Wire in the artificial plasma (exposition $160 \mathrm{~min}$.). 
on the chemical composition and the surface protection but also on the technology of processing that can be different according to the producer.

\section{Measuring system}

The developed original measuring system allows measurement of mechanical properties of stents, measurement of strain-stress curves or simulation of radial and axial deformation. We designed two arrangements of measuring systems, the measurement of the stent response to the axial and to the radial deformation. In case of radial deformation the device consists of hermetically closed plastic glass vessel in which the stent is placed in the distilled water or in the air. The stent is joined to the system of the tubes with the valves. The temperature of water can be held on constant value or its course can by defined by using the accurate thermostat with cooling for fast response. The tubes can be closed or opened by the valves so that the defined pressure of peristaltic pump affects the wall of stent. The expansion and the compression of the stent can be simulated in this way. Pressure course and corresponding change of stent radius are on-line measured by the probes connected to the computer. The original contactless method of measurement of the stent radius is based on the on-line processing of the digitalized image from the video camera (Topica TP $606 \mathrm{D} / 3$ ) by using the NI program. The axial prolongation of the stent is measured by the accurate linear potentiometer (position sensor) that is directly bounded with the screw of the positioning system that is used for longitudinal deformation. The axial force for longitudinal deformation is measured by the dynamometer that is connected directly to the positioning device. All measurements are on line controlled and processed by the LabVIEW software and hardware platform. The schematic diagrams of the measuring system in the arrangement for the measurement of radial forces, dynamic measurement and simula- tion and in the arrangement for axial (longitudinal) forces measurement are given in the figures 2 .

\section{Method of measurement}

The mechanical response of selfexpandable spiral Nitinol stents of the same design and different geometry was compared. The spiral stents are knitted from one Nitinol wire and can be described as a set of horizontally and vertically shifted spirals (springs). Measured stents differ in the diameter of Nitinol wire and in the rising of the winding. Number of single left and right spirals (number of the left and the right arms of stent) is common. We measured and compared stents, which differed only in one from above mentioned two parameters. From the practical point of view it is technically simpler to generate the axial force for deformation of stent instead of the radial force. It is very important from this reason to know the relation between the effect of radial and axial forces. We suppose for measurement that the result deformation doesn't depend on the type of functioning force (axial or radial) or that the relation between radial and axial forces effect is exactly given. This assumption we can verify experimentally by measurement of both arrangements of measuring device (see figure 2). All parameters of stents (diameter, length, temperature, axial force) are measured on-line simultaneously and repeatedly with given sampling frequency (typically $1 \mathrm{~s}$ ). Nitinol stent follows some properties, which correspond with the exclusive behavior of Nitinol, especially its superelasticity (3). Nitinol can exist in two phases, soft martensite for the lower temperature and hard austenite for the higher temperature. The temperature of transformation is given not only by the composition but also by the stress of stent. We must also study the relation between the strain-stress curves course and temperature from these reasons. All measurements were done for the laboratory and the body temperature.

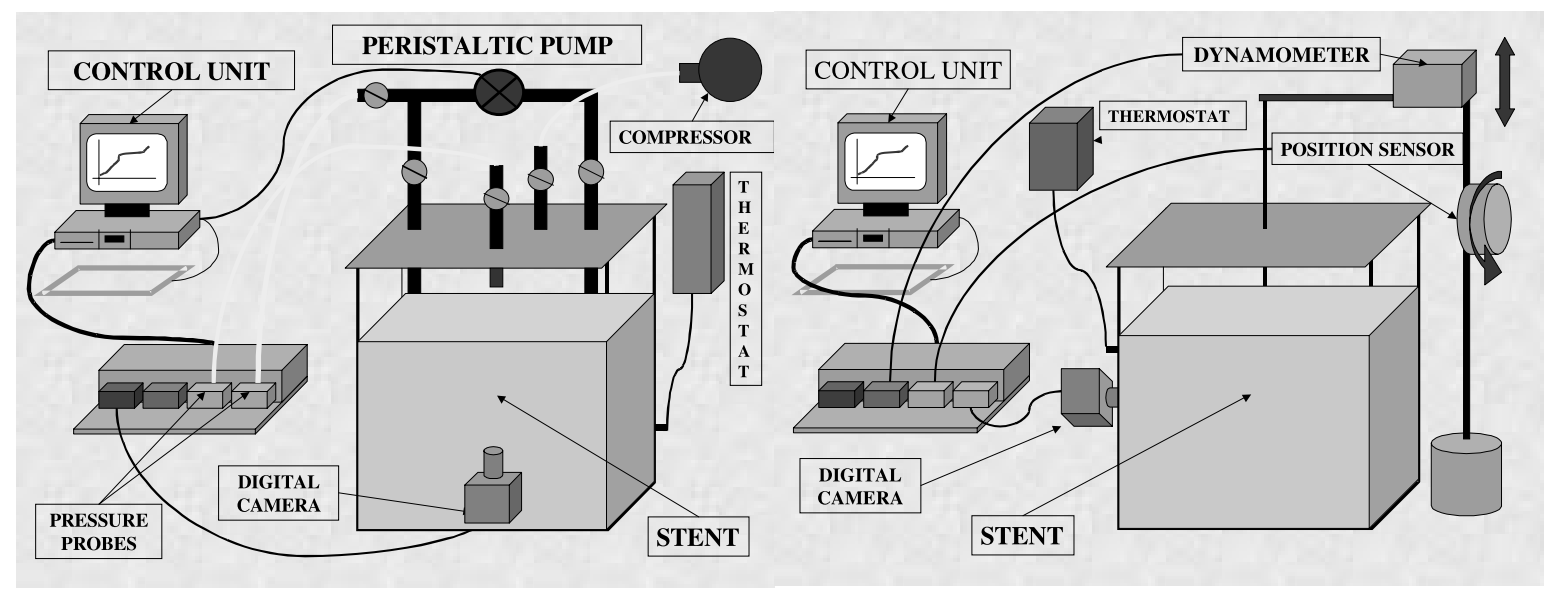

Fig. 2: Measuring system (a - arrangement for measurement of deformation by radial force, $\mathrm{b}$ - arrangement for measurement of deformation by axial force). 


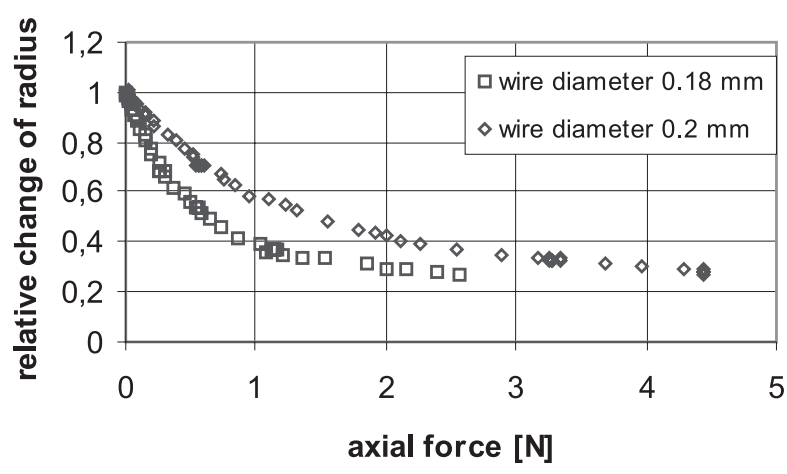

Fig. 3: Strain-stress curves of Nitinol stent for different wire diameter. The effect of axial force to radial deformation is illustrated.

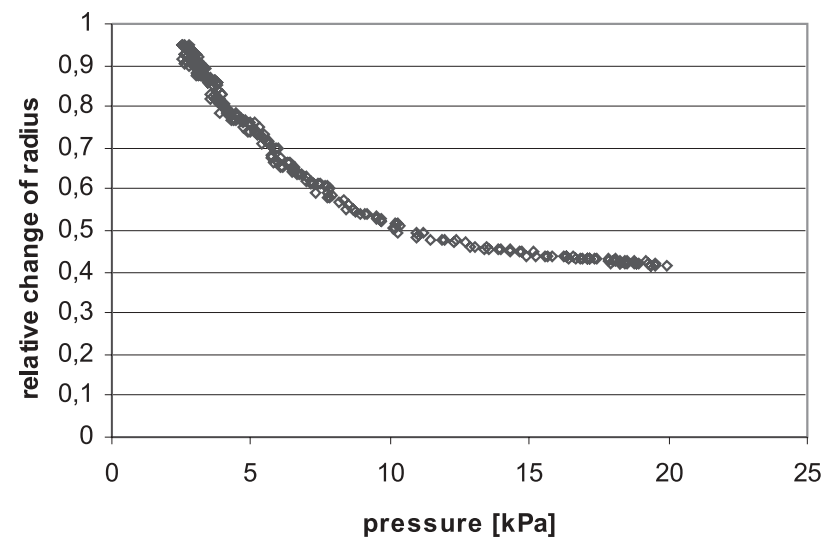

Fig. 5: Strain-stress curve of Nitinol stent. Effect of radial force (pressure) to radial deformation is illustrated.

\section{Results of measurement}

We compared the results of measurement of Nitinol stents with these parameters:

Diameter of Nitinol wire: 0.2 and $0.18 \mathrm{~mm}$

Number of left and right arms of stents: 12 left arms + 12 right arms (arm = single spiral)

Rising of winding: $25 \mathrm{~mm}$ and $28 \mathrm{~mm}$ (distance between successive coils of the single spring)

Geometry of stents: diameter $16 \mathrm{~mm}$, length $72 \mathrm{~mm}$ or diameter $12 \mathrm{~mm}$, length $77 \mathrm{~mm}$

Temperature of environment: laboratory temperature (approx. $20^{\circ} \mathrm{C}$ ) and body temperature $37^{\circ} \mathrm{C}$

We combined for measurements pairs of stents that differ only in one parameter, in diameter of Nitinol wire or in the rising of windings. Absolute value of diameter and length of the stents are not important because the deformation of the stent can be expressed as the relative change of the original size. The figure 3 illustrates the effect of axial force to the radial deformation of the stents with different diameter of Nitinol wire. The figure 4 illustrates the same type of de-

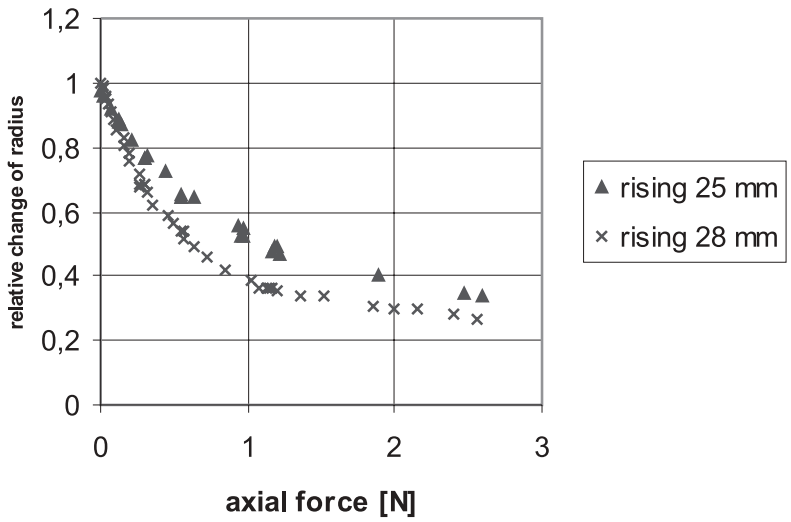

Fig. 4: Strain-stress curves of Nitinol wire for different rising of windings. The effect of axial force to radial deformation is illustrated.

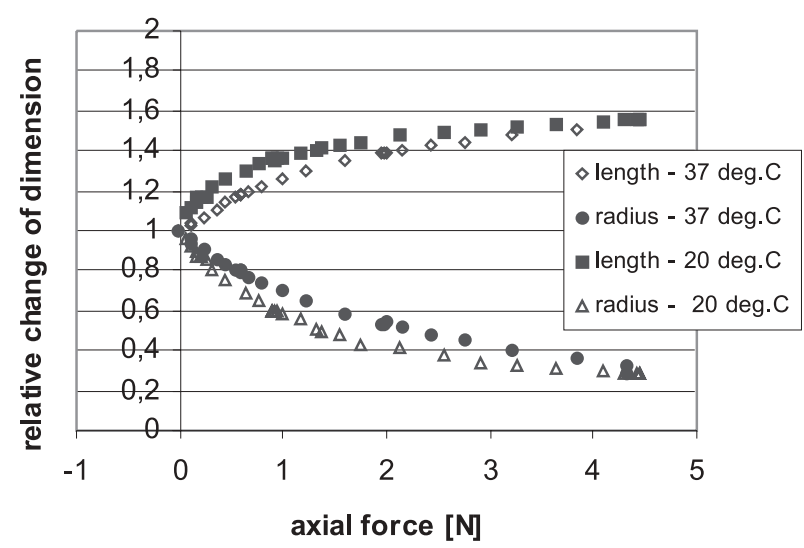

Fig. 6: Strain-stress curve of Nitinol stent. Effect of the temperature to the radial and axial deformation is illustrated.

formation for two stents with different rising of windings. The figure 5 illustrates the relative radial deformation of the stent caused by the radial force (outside pressure) and at last the figure 6 describes the influence of the temperature to the radial and axial deformation of stent.

\section{Conclusion}

The originally developed measuring system and methodology were successfully tested for measurement of mechanical properties of Nitinol selfexpandable stents. System allows according to its arrangement the generation of defined course of axial and radial forces functioning to the stent or biological system. The set of sensors and probes allows the accurate contactless measurement of strain; it means the radial and the axial deformation of stressed system. The experiments cleared some mechanical characteristics of tested stents and also confirmed the theoretical assumptions concerning the relation between the geometry and the construction of stent and its mechanical performance. For instance the relation between the radius and 
length changes enables us to estimate exactly the real length of stent after its pushing out from the delivery system and its expansion into the vessel. The measurements also confirmed theoretical assumption $(4,5)$ that the suitable choice of the diameter of wire is the best way of setting of required rigidity of the stent because the spring stiffness is directly proportional to fourth power of the diameter of the wire. It is necessary to accent on the opposite side that the diameter of the wire limits minimal size of the delivery system.

\section{References}

1. Duda SH. Physical properties of Endovascular Stents: An Experimental Comparison. J Vasc Intervent Radiol 2000 ;11:645-54.
2. Dyet JF, Watts WG, Ettles DF. Mechanical properties of metallic stents: How do these properties influence the choice of stent for specific lesions? CardioVasc Intervent Radiol 2000;23:47-54.

3. Shabalovskaya SA. On the nature of the biocompatibility and on medical applications of NiTi shape memory and superelastic alloys. Biomed Mater Eng 199;6(4):267-89.

4. www.efunda.com/designstandards/springs/

5. physics.uwstout.edu/statStr/Strength/Columns/cols 75

Doc. Ing. Josef Hanuš, CSc.,

Charles University in Prague, Faculty of Medicine in Hradec Králové, Department of Medical Biophysics, Šimkova 870, 50038 Hradec Králové, Czech Republic. e-mail: Hanus@lfhk.cuni.cz 